\title{
BENEFIT FROM SOLAR RADIATION AS ALTERNATIVE ENERGY IN RIYADH REGION IN SAUDI ARABIA
}

\section{Motirh Kueetm Al-Mutairi}

Associate professor of geography and climate, Department of Geography, Faculty of Arts, Princess Nourah bint Abdulrahman University, dr.motirh@hotmail.com, Mobile:00966-541167114

\begin{abstract}
The studied sites in the Riyadh region are characterized by a great abundance of natural and human resources throughout the year. So, they are qualified to invest solar energy as one of the most important sources of natural alternative energy, due to the abundance of solar radiation regularly during the year and its high intensity especially in the summer. This season is the period of the maximum demand for electric power.

Solar energy is regularly available in the Riyadh area during 4380 hours of solar brightness per year at the studied sites. The solar energy of the Riyadh region during this period can provide a huge potential for electricity production with the renewable energy technologies and projects. So, the alternative solar energy can be required without high costs compared to the traditional energy resources draining most state budget and causing the large environment pollution.

The production of electricity as an alternative renewable energy resource to traditional energy resources is one of the priorities of the future projects in the Kingdom of Saudi Arabia, which seeks to diversify its sources of energy by providing the maximum of the alternative energy. This alternative option has become the priority of sustainable development, which aims to preserve human and natural resources with reducing the electricity production cost. This option make possible the gradual absorption of traditional energy sources polluting the environment and threatened with depletion.
\end{abstract}

Keywords: Solar Radiation, Renewable Energy, Solar Angles, Horizontal Surface, Titled Surface, Riyadh Region, Saudi Arabia.

\section{1- INTRODUCTION}

Since the 1980s, solar energy has become a global interest because it is the most important source of alternative renewable energy to the traditional sources of energy which are shrinking and disappearing (oil, natural gas, etc...).

Solar energy is a regular energy source can be converted using many technologies with low costs comparing to electricity energy. The world interest is increasingly in exploiting solar energy with collect and store, because it is natural and clean energy. Access to the solar energy exploitation as a source of renewable energy in many applications like industrial and agricultural consumption requires clarification of the solar radiation system on the Earth's surface.

The solar radiation of the Earth's surface is the product of the helium atoms formed by the fusion of hydrogen atoms. The formation of a single helium atom requires the fusion of two hydrogen atoms with a mass greater than the helium atom. The difference between the missing mass of the fusion process products the radioactive energy, which is the solar energy emitting into the exterior sun sphere and reaches via the atmosphere the Earth's surface.

The availability of alternative energy sources, especially solar energy, will contribute to reducing the dependence of the national economy on oil resources and to increasing employment opportunities in large labor numbers in alternative energy sectors such as the solar energy sector.

This study aims to examine the possibility of producing electricity as an alternative, clean and renewable energy resource by solar radiation in the favorable climatic conditions of the Riyadh region. Therefore, this study is of environmental, economic and social interest and it is the important step in the investment of solar energy as a natural, clean, regularly available energy, which can produce an alternative electric energy with low costs 
compared to conventional energy production costs.

\section{STUDY AREA:}

The studied sites are located in Riyadh, AlDera'iyah and Al-Kharj, between the latitudes $\mathrm{E} 20^{\circ} 09^{\prime}$ and $\mathrm{E} 24^{\circ} 45^{\prime}$ and the longitudes $\mathrm{N} 46^{\circ} 32^{\prime}$ and $\mathrm{N} 47^{\circ} 18^{\prime}$. The time limits of this study in the number of days of the year used in calculating the average daily amount of solar radiation at midday $(12 \mathrm{pm})$ in each of the study sites (Table 1 and Figure 1).

Tab. 1: Coordinates of studied sites.

\begin{tabular}{|c|c|c|c|}
\hline Site & $\begin{array}{c}\text { Longitude } \\
(\mathbf{E})\end{array}$ & $\begin{array}{c}\text { Latitude } \\
(\mathbf{N})\end{array}$ & $\begin{array}{c}\text { Elevation } \\
(\mathbf{m})\end{array}$ \\
\hline Riyadh & $46^{\circ} 43^{\prime}$ & $24^{\circ} 34^{\prime}$ & 564 \\
\hline Al-Dera'iyah & $46^{\circ} 32^{\prime}$ & $24^{\circ} 45^{\prime}$ & 701 \\
\hline Al-Kharj & $47^{\circ} 18^{\prime}$ & $24^{\circ} 09^{\prime}$ & 435 \\
\hline
\end{tabular}

\section{METHODOLOGY:}

This research is based on the application of the modern model developed by Ashrae using a series of methodological steps to calculate the global solar radiation $\left(\mathrm{I}_{\mathrm{T}}\right)$ reaching the Earth's surface. This radiation is composed by the direct radiation $\left(I_{D}\right)$, diffuse sky radiation $\left(I_{d}\right)$, and refracted ground radiation $\left(\mathrm{I}_{\mathrm{r}}\right)$. So, the global solar radiation in this model is estimated by the following equation: (Ashrae, 2011)

$$
I_{T}=I_{D}+I_{d}+I_{r}\left(W / h / m^{2}\right)
$$

Calculating the global of falling rays on horizontal, titled or vertical surfaces during sunrise to sunset hours requires the following methodological steps:

\subsection{Total direct radiation:}

The total direct solar radiation is directly related to two factors; the direct and vertical radiation $\left(\mathrm{I}_{\mathrm{DN}}\right)$ and the solar incident angle $(\theta)$. It is calculated by the following equation: (Ashrae, 2011)

with:

$$
I_{D}=I_{D N} \operatorname{Cos}(\theta)\left(W / h / m^{2}\right)
$$

$(\theta)$ : solar incident angle.

$\mathrm{I}_{\mathrm{DN}}$ : direct and vertical solar radiation $\left(\mathrm{W} / \mathrm{h} / \mathrm{m}^{2}\right)$.

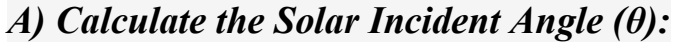

The solar incident angle is associated with several other angles of solar radiation. It is calculated by a series of equations as follows: (Duffie and Backman, 2013)

$$
\begin{gathered}
\theta=\operatorname{arcCos}\left[\operatorname{Cos}(\psi) \operatorname{Cos}\left(\gamma_{\mathrm{s}}\right) \operatorname{Sin}(\beta)+\operatorname{Sin}\right. \\
(\psi) \operatorname{Cos}(\beta)]
\end{gathered}
$$

The $\beta$ represents the optimum tilt angle of the surface and can be calculated by the following equation:

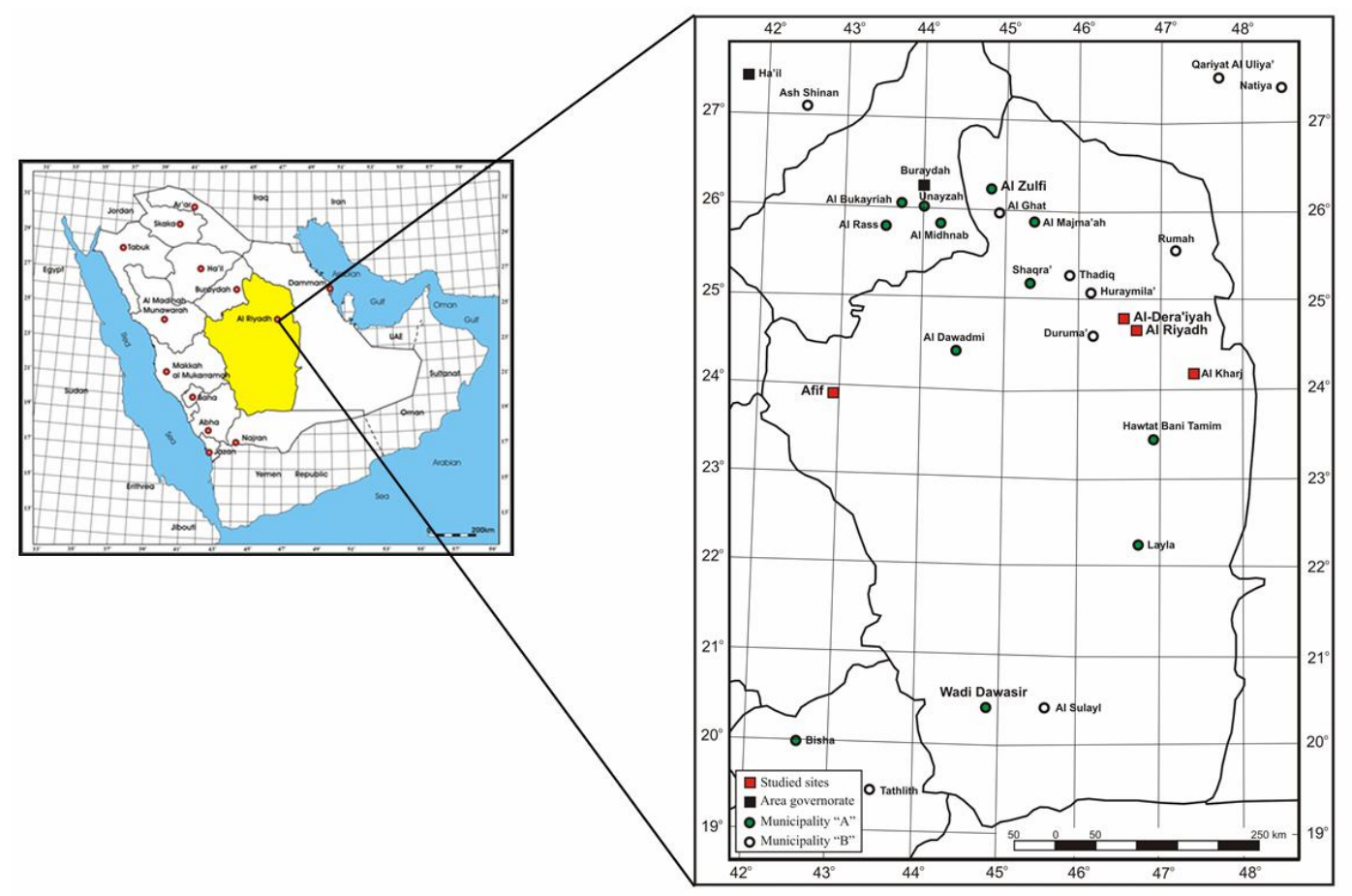

Fig. 1: Geographic location of studied sites. 
$\beta=\operatorname{arcCos}[\operatorname{Cos}(\phi) \operatorname{Cos}(\delta) \operatorname{Cos}(\omega)+\operatorname{Sin}(\phi)$ $\operatorname{Sin}(\delta)]$

$\beta=90^{\circ}$ for the vertical surface and $\beta=0^{\circ}$ for the horizontal surface horizontal. $\Psi$ is the Solar Altitude Angle. It can be calculated by the following equation:

$$
\psi=\operatorname{arcSin}[\operatorname{Cos}(\phi) \operatorname{Cos}(\delta) \operatorname{Sin}(\omega)+\operatorname{Sin}(\phi)
$$$$
\operatorname{Cos}(\delta)]
$$

with:

$\phi$ : Latitude Angle.

$\delta$ The sun declination angle. It equals:

$\delta=23.45^{\circ}$ Sin $[0.9863(284+\mathrm{N})]$ (Cooper, 1969)

with $\mathrm{N}$ : the Julian number of the day.

$\omega$ : Solar Time Angle. It equals to:

$\omega=15$ (LAT-12) (Messenger and Vente, 2003)

The LAT is the Local Time of Local Time.

$\gamma_{\mathrm{s}}$ : Solar Azimuth Angle. It is calculated as follows:

$$
\gamma_{\mathrm{s}}=\operatorname{arcSin}\left[\frac{\operatorname{Cos}(\delta) \operatorname{Sin}(\omega)}{\operatorname{Cos}(\psi)}\right]
$$

\section{B) Direct Normal Solar Radiation Intensity $\left(I_{D N)}\right.$ :}

The direct Normal Solar Radiation Intensity can be calculated using three variables as:

A- Visible solar radiation when air mass is equal to 0 . It can be calculated by the following equation:

$\mathrm{A}=1147.59+57.5 \operatorname{Sin}(0.01743 \mathrm{~N}+1.47822)$

B- Weather eclipse coefficient is calculated by the following equation:

$$
\mathrm{B}=0.16395+0.0237 \mathrm{Sin}(0.02017 \mathrm{~N}+
$$

C- $\Psi$ is the solar altitude angle.

So, the direct and vertical solar radiation is equal to: (Ashrae, 2011)

$$
\mathrm{I}_{\mathrm{DN}}=\frac{\mathrm{A}}{\exp \left[\frac{\mathrm{B}}{\operatorname{Sin}(\boldsymbol{\Psi})}\right]}
$$

2.2 Solar radiation flux incident on horizontal surface $\left(\mathbf{I}_{\mathrm{DH}}\right)$ :

This solar radiation is calculated by the following equation: (Ashrae, 2011)

$$
\begin{aligned}
& \mathrm{I}_{\mathrm{DH}}=\mathrm{I}_{\mathrm{DN}} \operatorname{Cos}\left(\theta_{\mathrm{H}}\right)\left(\mathrm{W} / \mathrm{h} / \mathrm{m}^{2}\right) \\
& \mathrm{I}_{\mathrm{DH}}=\mathrm{I}_{\mathrm{DN}} \operatorname{Sin}(\psi)\left(\mathrm{W} / \mathrm{h} / \mathrm{m}^{2}\right)
\end{aligned}
$$

2.3 Solar radiation flux incident on vertical surface $\left(\mathbf{I}_{\mathrm{DV}}\right)$ :

This solar radiation is calculated by the following equation: (Ashrae, 2011)

$$
\begin{gathered}
\mathrm{I}_{\mathrm{DV}}=\mathrm{I}_{\mathrm{DN}} \operatorname{Cos}\left(\theta_{\mathrm{V}}\right)\left(\mathrm{W} / \mathrm{h} / \mathrm{m}^{2}\right) \\
\mathrm{I}_{\mathrm{DV}}=\mathrm{I}_{\mathrm{DN}} \operatorname{Cos}(\psi) \operatorname{Cos}\left(\gamma_{\mathrm{s}}\right)\left(\mathrm{W} / \mathrm{h} / \mathrm{m}^{2}\right)
\end{gathered}
$$

\subsection{Diffuse solar radiation from a clear sky $\left(\mathbf{I}_{d}\right)$ :}

This solar radiation is calculated by the following equation: (Ashrae, 2011)

$$
\mathrm{I}_{\mathrm{d}}=\mathrm{C} \mathrm{I}_{\mathrm{DN}} \mathrm{F}_{\mathrm{ss}}\left(\mathrm{W} / \mathrm{h} / \mathrm{m}^{2}\right)
$$

With $\mathrm{C}$ as diffuse solar radiation coefficient. It equals to:

$$
\mathrm{C}=0.1207+0.0179 \operatorname{Sin}(0.0203 \mathrm{~N}+3.9799)
$$

Fss is the diffuse conversion factor, given as:

$$
\mathrm{F}_{\mathrm{ss}}=\frac{1+\operatorname{Cos}(\beta)}{2}
$$

\subsection{Diffuse solar radiation reflected from the ground surface $\left(I_{r}\right)$ :}

This solar radiation is calculated by the following equation: (ASHRAE, 2011)

$$
I_{\mathrm{r}}=I_{\mathrm{TH} \rho} \mathrm{F}_{\mathrm{sg}}\left(\mathrm{W} / \mathrm{h} / \mathrm{m}^{2}\right)
$$

with $\rho$ the surface reflection coefficient. It equals to 0.2 for the normal or agricultural surface and 0.8 for the snow-covered surface (Lunde, 1980).

$$
\mathrm{F}_{\mathrm{sg}}=\frac{1-\operatorname{Cos}(\beta)}{2}
$$

With $F_{s g}$ is the reflected conversion factor, given as:

$\mathrm{I}_{\mathrm{TH}}$ : Solar radiation on the surface, which is equal to :

$$
\begin{gathered}
\mathrm{I}_{\mathrm{TH}}=\mathrm{I}_{\mathrm{DN}}(\mathrm{C}+\operatorname{Sin} \psi)\left(\mathrm{W} / \mathrm{h} / \mathrm{m}^{2}\right) \\
\mathrm{I}_{\mathrm{TH}}=\mathrm{I}_{\mathrm{DN}}[\mathrm{C}+\operatorname{Sin}(\psi)]\left(\mathrm{W} / \mathrm{h} / \mathrm{m}^{2}\right)
\end{gathered}
$$

\subsection{Sunshine duration $(\mathrm{S})$ :}

The sunshine duration is the maximum number of hours of daylight brightness (day length). This period is related to the latitude and declination angles. It is given as: (Duffie and Beckman, 2013)

$$
\mathrm{S}=0.1333 \operatorname{arcCos}(-\tan \varnothing \tan \delta)
$$




\section{RESULTS AND DISCUSSION:}

The main results of this study are:

\subsection{Determining the factors affecting the utilization of solar radiation:}

The benefit of solar radiation is related to calculate the total direct and vertical solar radiation on the Earth's surface, solar incident angle and the number of solar brightness hours. This solar radiation on the titled, horizontal and vertical surfaces helps to select the best locations for solar cell panels producing electricity by solar radiation. The location of solar cell panels is related to three main factors affecting its electric energy production:

\section{A) Determining the direction of solar cell panels:}

Solar cell panels are oriented South if they are located in the Northern hemisphere and South if they are located in the Northern hemisphere. Due to the location of the study area in the Northern hemisphere, solar cell panels are heading South.

\section{B) Determination of the slope of the surface of the solar cell panels:}

$$
\beta=(0.84 \phi)+1^{\circ}
$$

The location of the place is related to the titled angle of the of the solar cell panels surface. So, the best angle for the solar cell during the year of the sites located in latitudes less than $25^{\circ}$ is given by the following equation:

$$
\beta=(0.84 \phi)+1^{\mathrm{o}}
$$

\section{C) Calculate the solar incident angle ( $\theta)$ :}

The solar incident angle is associated with three angles, the optimum tilt angle of the surface $(\beta)$, the solar altitude angle $(\psi)$ and the solar azimuth angle $\left(\gamma_{\mathrm{s})}\right.$. The solar incident angle of the studied sites was calculated daily at 12 o'clock to determine the average daily solar radiation at the studied sites was calculated daily at 12 o'clock to middle of each month as shown in Tables 2, 3 and 4.

This angle variations depend on the time, the latitude of the site and the sun declination angle. So, the solar radiation reaches the maximum if this angle is vertical or close to $90^{\circ}$, because the distance traveled by solar radiation and its reflectance and dispersion become less. The solar radiation rises gradually after sunrise with the solar altitude angle rising to peak at noon. The sun is vertical on the surface of the earth at noon, and gradually decrease after noon until sunset.

\begin{tabular}{|c|c|c|c|c|c|c|c|c|c|c|c|c|}
\hline \multirow{2}{*}{ 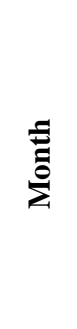 } & 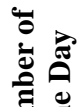 & \multicolumn{5}{|c|}{ Solar angles } & \multicolumn{3}{|c|}{$\beta$ angle } & \multicolumn{3}{|c|}{$\theta$ angle } \\
\hline & $\mathbf{n}$ & $\Phi$ & $\gamma_{\mathrm{s}}$ & $\mathbf{\Omega}$ & $\psi$ & $\boldsymbol{\delta}$ & 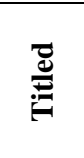 & 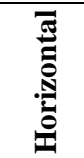 & 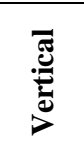 & $\stackrel{\vec{D}}{E}$ & 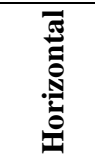 & 胥 \\
\hline Jan. & 15 & $24.15^{\circ}$ & $0.0^{\circ}$ & $0.0^{\circ}$ & $44.6^{\circ}$ & $-21.3^{\circ}$ & $22.0^{\circ}$ & $0.0^{\circ}$ & $90.0^{\circ}$ & $23.4^{\circ}$ & $45.4^{\circ}$ & $44.6^{\circ}$ \\
\hline Feb. & 46 & $24.15^{\circ}$ & $0.0^{\circ}$ & $0.0^{\circ}$ & $52.6^{\circ}$ & $-13.3^{\circ}$ & $22.0^{\circ}$ & $0.0^{\circ}$ & $90.0^{\circ}$ & $15.4^{\circ}$ & $37.4^{\circ}$ & $52.6^{\circ}$ \\
\hline Mar. & 74 & $24.15^{\circ}$ & $0.0^{\circ}$ & $0.0^{\circ}$ & $63.0^{\circ}$ & $-02.8^{\circ}$ & $22.0^{\circ}$ & $0.0^{\circ}$ & $90.0^{\circ}$ & $05.0^{\circ}$ & $27.0^{\circ}$ & $63.0^{\circ}$ \\
\hline Apr. & 105 & $24.15^{\circ}$ & $0.0^{\circ}$ & $0.0^{\circ}$ & $75.3^{\circ}$ & $09.4^{\circ}$ & $22.0^{\circ}$ & $0.0^{\circ}$ & $90.0^{\circ}$ & $07.3^{\circ}$ & $14.7^{\circ}$ & $75.3^{\circ}$ \\
\hline May & 135 & $24.15^{\circ}$ & $0.0^{\circ}$ & $0.0^{\circ}$ & $84.6^{\circ}$ & $18.8^{\circ}$ & $22.0^{\circ}$ & $0.0^{\circ}$ & $90.0^{\circ}$ & $16.6^{\circ}$ & $05.4^{\circ}$ & $84.6^{\circ}$ \\
\hline June & 166 & $24.15^{\circ}$ & $0.0^{\circ}$ & $0.0^{\circ}$ & $89.2^{\circ}$ & $23.3^{\circ}$ & $22.0^{\circ}$ & $0.0^{\circ}$ & $90.0^{\circ}$ & $21.2^{\circ}$ & $00.8^{\circ}$ & $89.2^{\circ}$ \\
\hline Jul. & 196 & $24.15^{\circ}$ & $0.0^{\circ}$ & $0.0^{\circ}$ & $87.4^{\circ}$ & $21.5^{\circ}$ & $22.0^{\circ}$ & $0.0^{\circ}$ & $90.0^{\circ}$ & $19.4^{\circ}$ & $02.6^{\circ}$ & $87.4^{\circ}$ \\
\hline Aug. & 227 & $24.15^{\circ}$ & $0.0^{\circ}$ & $0.0^{\circ}$ & $79.6^{\circ}$ & $13.8^{\circ}$ & $22.0^{\circ}$ & $0.0^{\circ}$ & $90.0^{\circ}$ & $11.6^{\circ}$ & $10.4^{\circ}$ & $79.6^{\circ}$ \\
\hline Sep. & 258 & $24.15^{\circ}$ & $0.0^{\circ}$ & $0.0^{\circ}$ & $68.1^{\circ}$ & $02.2^{\circ}$ & $22.0^{\circ}$ & $0.0^{\circ}$ & $90.0^{\circ}$ & $00.1^{\circ}$ & $21.9^{\circ}$ & $68.1^{\circ}$ \\
\hline Oct. & 288 & $24.15^{\circ}$ & $0.0^{\circ}$ & $0.0^{\circ}$ & $56.3^{\circ}$ & $-09.6^{\circ}$ & $22.0^{\circ}$ & $0.0^{\circ}$ & $90.0^{\circ}$ & $11.7^{\circ}$ & $33.7^{\circ}$ & $56.3^{\circ}$ \\
\hline Nov. & 319 & $24.15^{\circ}$ & $0.0^{\circ}$ & $0.0^{\circ}$ & $46.7^{\circ}$ & $-19.1^{\circ}$ & $22.0^{\circ}$ & $0.0^{\circ}$ & $90.0^{\circ}$ & $21.3^{\circ}$ & $43.3^{\circ}$ & $46.7^{\circ}$ \\
\hline Dec. & 349 & $24.15^{\circ}$ & $0.0^{\circ}$ & $0.0^{\circ}$ & $42.5^{\circ}$ & $-23.3^{\circ}$ & $22.0^{\circ}$ & $0.0^{\circ}$ & $90.0^{\circ}$ & $25.5^{\circ}$ & $47.5^{\circ}$ & $42.5^{\circ}$ \\
\hline
\end{tabular}

Tab. 2: Solar angles of Al-Kharj site. 
Tab. 3: Solar angles of Riyadh site.

\begin{tabular}{|c|c|c|c|c|c|c|c|c|c|c|c|c|}
\hline \multirow{2}{*}{ 䓂 } & 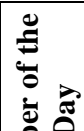 & \multicolumn{5}{|c|}{ Solar angles } & \multicolumn{3}{|c|}{$\beta$ angle } & \multicolumn{3}{|c|}{$\theta$ angle } \\
\hline & $\mathbf{n}$ & $\phi$ & $\gamma_{\mathrm{s}}$ & $\omega$ & $\psi$ & $\delta$ & 总 & 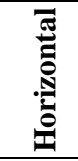 & 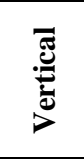 & 总 & 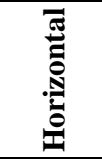 & : \\
\hline Jan. & 15 & $24.57^{\circ}$ & $0.0^{\circ}$ & $0.0^{\circ}$ & $44.6^{\circ}$ & $-21.3^{\circ}$ & $22.0^{\circ}$ & $0.0^{\circ}$ & $90.0^{\circ}$ & $23.8^{\circ}$ & $45.8^{\circ}$ & $44.2^{\circ}$ \\
\hline Feb. & 46 & $24.57^{\circ}$ & $0.0^{\circ}$ & $0.0^{\circ}$ & $52.6^{\circ}$ & -13.3 & $22.0^{\circ}$ & $0.0^{\circ}$ & $90.0^{\circ}$ & $15.9^{\circ}$ & $37.9^{\circ}$ & $52.1^{\circ}$ \\
\hline Mar. & 74 & $24.57^{\circ}$ & $0.0^{\circ}$ & $0.0^{\circ}$ & $63.0^{\circ}$ & $-02.8^{\circ}$ & $22.0^{\circ}$ & $0.0^{\circ}$ & $90.0^{\circ}$ & $5.4^{\circ}$ & $27.4^{\circ}$ & $62.6^{\circ}$ \\
\hline Apr. & 105 & $24.57^{\circ}$ & $0.0^{\circ}$ & $0.0^{\circ}$ & $75.3^{\circ}$ & $09.4^{\circ}$ & $22.0^{\circ}$ & $0.0^{\circ}$ & $90.0^{\circ}$ & $6.8^{\circ}$ & $15.2^{\circ}$ & $74.8^{\circ}$ \\
\hline May & 135 & $24.57^{\circ}$ & $0.0^{\circ}$ & $0.0^{\circ}$ & $84.6^{\circ}$ & $18.8^{\circ}$ & $22.0^{\circ}$ & $0.0^{\circ}$ & $90.0^{\circ}$ & $16.2^{\circ}$ & $05.8^{\circ}$ & $84.2^{\circ}$ \\
\hline June & 166 & $24.57^{\circ}$ & $0.0^{\circ}$ & $0.0^{\circ}$ & $89.2^{\circ}$ & $23.3^{\circ}$ & $22.0^{\circ}$ & $0.0^{\circ}$ & $90.0^{\circ}$ & $20.7^{\circ}$ & $01.3^{\circ}$ & $88.7^{\circ}$ \\
\hline Jul. & 196 & $24.57^{\circ}$ & $0.0^{\circ}$ & $0.0^{\circ}$ & $87.4^{\circ}$ & $21.5^{\circ}$ & $22.0^{\circ}$ & $0.0^{\circ}$ & $90.0^{\circ}$ & $19.0^{\circ}$ & $03.0^{\circ}$ & $87.0^{\circ}$ \\
\hline Aug. & 227 & $24.57^{\circ}$ & $0.0^{\circ}$ & $0.0^{\circ}$ & $79.6^{\circ}$ & $13.8^{\circ}$ & $22.0^{\circ}$ & $0.0^{\circ}$ & $90.0^{\circ}$ & $11.2^{\circ}$ & $10.8^{\circ}$ & $79.2^{\circ}$ \\
\hline Sep. & 258 & $24.57^{\circ}$ & $0.0^{\circ}$ & $0.0^{\circ}$ & $68.1^{\circ}$ & $02.2^{\circ}$ & $22.0^{\circ}$ & $0.0^{\circ}$ & $90.0^{\circ}$ & $0.3^{\circ}$ & $22.3^{\circ}$ & $67.7^{\circ}$ \\
\hline Oct. & 288 & $24.57^{\circ}$ & $0.0^{\circ}$ & $0.0^{\circ}$ & $56.3^{\circ}$ & $-09.6^{\circ}$ & $22.0^{\circ}$ & $0.0^{\circ}$ & $90.0^{\circ}$ & $12.2^{\circ}$ & $34.2^{\circ}$ & $55.8^{\circ}$ \\
\hline Nov. & 319 & $24.57^{\circ}$ & $0.0^{\circ}$ & $0.0^{\circ}$ & $46.7^{\circ}$ & $-19.1^{\circ}$ & $22.0^{\circ}$ & $0.0^{\circ}$ & $90.0^{\circ}$ & $21.7^{\circ}$ & $43.7^{\circ}$ & $46.3^{\circ}$ \\
\hline Dec. & 349 & $24.57^{\circ}$ & $0.0^{\circ}$ & $0.0^{\circ}$ & $42.5^{\circ}$ & $-23.3^{\circ}$ & $22.0^{\circ}$ & $0.0^{\circ}$ & $90.0^{\circ}$ & $25.9^{\circ}$ & $47.9^{\circ}$ & $42.1^{\circ}$ \\
\hline
\end{tabular}

Tab. 4: Solar angles of Al-Dera'iyah site.

\begin{tabular}{|c|c|c|c|c|c|c|c|c|c|c|c|c|}
\hline \multirow{2}{*}{ 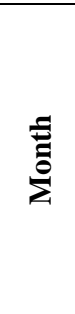 } & : & \multicolumn{5}{|c|}{ Solar angles } & \multicolumn{3}{|c|}{$\beta$ angle } & \multicolumn{3}{|c|}{$\theta$ angle } \\
\hline & $\mathbf{n}$ & $\phi$ & $\gamma_{s}$ & $\omega$ & $\psi$ & $\delta$ & 总 & 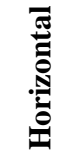 & 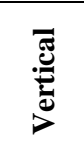 & 总 & 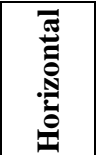 & : \\
\hline Jan. & 15 & $24.57^{\circ}$ & $0.0^{\circ}$ & $0.0^{\circ}$ & $44.6^{\circ}$ & $-21.3^{\circ}$ & $22.0^{\circ}$ & $0.0^{\circ}$ & $90.0^{\circ}$ & $24.0^{\circ}$ & $46.0^{\circ}$ & $44.0^{\circ}$ \\
\hline Feb. & 46 & $24.57^{\circ}$ & $0.0^{\circ}$ & $0.0^{\circ}$ & $52.6^{\circ}$ & $-13.3^{\circ}$ & $22.0^{\circ}$ & $0.0^{\circ}$ & $90.0^{\circ}$ & $16.0^{\circ}$ & $38.0^{\circ}$ & $52.0^{\circ}$ \\
\hline Mar. & 74 & $24.57^{\circ}$ & $0.0^{\circ}$ & $0.0^{\circ}$ & $63.0^{\circ}$ & $-02.8^{\circ}$ & $22.0^{\circ}$ & $0.0^{\circ}$ & $90.0^{\circ}$ & $5.6^{\circ}$ & $27.6^{\circ}$ & $62.4^{\circ}$ \\
\hline Apr. & 105 & $24.57^{\circ}$ & $0.0^{\circ}$ & $0.0^{\circ}$ & $75.3^{\circ}$ & $09.4^{\circ}$ & $22.0^{\circ}$ & $0.0^{\circ}$ & $90.0^{\circ}$ & $6.7^{\circ}$ & $15.3^{\circ}$ & $74.7^{\circ}$ \\
\hline May & 135 & $24.57^{\circ}$ & $0.0^{\circ}$ & $0.0^{\circ}$ & $84.6^{\circ}$ & $18.8^{\circ}$ & $22.0^{\circ}$ & $0.0^{\circ}$ & $90.0^{\circ}$ & $16.0^{\circ}$ & $6.0^{\circ}$ & $84.0^{\circ}$ \\
\hline June & 166 & $24.57^{\circ}$ & $0.0^{\circ}$ & $0.0^{\circ}$ & $89.2^{\circ}$ & $23.3^{\circ}$ & $22.0^{\circ}$ & $0.0^{\circ}$ & $90.0^{\circ}$ & $20.6^{\circ}$ & $1.4^{\circ}$ & $88.6^{\circ}$ \\
\hline Jul. & 196 & $24.57^{\circ}$ & $0.0^{\circ}$ & $0.0^{\circ}$ & $87.4^{\circ}$ & $21.5^{\circ}$ & $22.0^{\circ}$ & $0.0^{\circ}$ & $90.0^{\circ}$ & $18.8^{\circ}$ & $3.2^{\circ}$ & $86.8^{\circ}$ \\
\hline Aug. & 227 & $24.57^{\circ}$ & $0.0^{\circ}$ & $0.0^{\circ}$ & $79.6^{\circ}$ & $13.8^{\circ}$ & $22.0^{\circ}$ & $0.0^{\circ}$ & $90.0^{\circ}$ & $11.0^{\circ}$ & $11.0^{\circ}$ & $79.0^{\circ}$ \\
\hline Sep. & 258 & $24.57^{\circ}$ & $0.0^{\circ}$ & $0.0^{\circ}$ & $68.1^{\circ}$ & $02.2^{\circ}$ & $22.0^{\circ}$ & $0.0^{\circ}$ & $90.0^{\circ}$ & $0.5^{\circ}$ & $22.5^{\circ}$ & $67.5^{\circ}$ \\
\hline Oct. & 288 & $24.57^{\circ}$ & $0.0^{\circ}$ & $0.0^{\circ}$ & $56.3^{\circ}$ & $-09.6^{0}$ & $22.0^{\circ}$ & $0.0^{\circ}$ & $90.0^{\circ}$ & $12.3^{\circ}$ & $34.3^{\circ}$ & $55.7^{\circ}$ \\
\hline Nov. & 319 & $24.57^{\circ}$ & $0.0^{\circ}$ & $0.0^{\circ}$ & $46.7^{\circ}$ & $-19.1^{\circ}$ & $22.0^{\circ}$ & $0.0^{\circ}$ & $90.0^{\circ}$ & $21.9^{\circ}$ & $43.9^{\circ}$ & $46.1^{\circ}$ \\
\hline Dec. & 349 & $24.57^{\circ}$ & $0.0^{\circ}$ & $0.0^{\circ}$ & $42.5^{\circ}$ & $-23.3^{\circ}$ & $22.0^{\circ}$ & $0.0^{\circ}$ & $90.0^{\circ}$ & $26.1^{\circ}$ & $48.1^{\circ}$ & $41.9^{\circ}$ \\
\hline
\end{tabular}

The declination angle increases gradually from April 12 from $8.3^{\circ}$ to a maximum of $23.4^{\circ}$ during the period from June 16 to 26 , which includes the summer solstice period and then decreases gradually to reach $-23.4^{\circ}$ during the period From 16 to 26 December, which includes the winter solstice. However, this angle reaches $-1.0^{\circ}$ on September 23 (autumn equinox) and $-0.8^{\circ}$ during the 20th of March (Equinox Spring).

The solar altitude angle is also increasing from $42.4^{\circ}$ during the period from 17 to 24 December, which includes the winter solstice and a maximum of $89.3^{\circ}$ during the period from June 18 to 25 , which includes the summer solstice. Also, this angle is $64.8^{\circ}$ on September 23 (autumn equinox) and $65.0^{\circ}$ on March 20 (Spring Equinox).

Thus, the average solar incident angle for horizontal surfaces ranges between $0.8^{\circ}$ and $47.5^{\circ}$ at Al-Kharj site and between $1.3^{\circ}$ and $47.9^{\circ}$ at Riyadh site and between $1.4^{\circ}$ and $48.1^{\circ}$ at Al-Dera'iyah site during June and July, respectively. For the titled surfaces, the mean solar incident angle is ranged between $0.1^{\circ}$ and $25.5^{\circ}$ at Al-Kharj site, between $0.3^{\circ}$ and $25.9^{\circ}$ at 
Riyadh site and between $0.5^{\circ}$ and $26.1^{\circ}$ at AlDera'iyah site during September and December respectively. For the vertical surfaces, the average of solar incident angle is ranging between $42.5^{\circ}$ and $85.2^{\circ}$ at Al-Kharj site and between $42.1^{\circ}$ and $88.7^{\circ}$ at Riyadh site and between $41.9^{\circ}$ and $88.6^{\circ}$ at Al-Dera'iyah site during December and June, respectively.

\section{D) Determining the insolation duration:}

The insolation duration is the period between the sunrise and sunset. The solar radiation is related to the duration of solar brightness during the day. So, the solar radiation increases as the insolation duration as is greater.

The region of Riyadh is characterized by a sunny, cloud-free atmosphere during the year with an average sunrise ranging between 10.5 and 13.5 hours/day (Tab. 5, 6 and 7). The duration insolation is long and affects directly the solar radiation falling on the surface in Riyadh. This solar radiation contributes regularly to the provision of sustainable energy during the year. Counter to the solar altitude angle, the insolation duration appears more homogenous in its spatial distribution as showing the average duration of sunrise, which ranges from 10.7 to 11.0 hours during the winter, from 12.5 and 12.6 hours during the spring, from 13.0 and 13.3 hours during the summer and from 11.4 and 11.5 hours during autumn (Fig. 2).

Tab. 5: The radiation flux incident at 12 noon on different surfaces in Al-Dera'iyah site.

\begin{tabular}{|c|c|c|c|c|c|}
\hline \multirow{2}{*}{ Month } & \multirow{2}{*}{$\mathbf{S}(\mathbf{h r})$} & \multicolumn{4}{|c|}{ Solar radiation flux $\left(\mathbf{W h r} / \mathbf{m}^{2}\right)$} \\
\cline { 3 - 6 } & & $\mathbf{I}_{\mathbf{D N}}$ & $\mathbf{I}_{\mathbf{D}}$ & $\mathbf{I}_{\mathbf{D H}}$ & $\mathbf{I}_{\mathbf{D V}}$ \\
\hline Jan. & 10.6 & 905.3 & 826.9 & 628.7 & 651.4 \\
\hline Feb. & 11.2 & 931.4 & 895.1 & 733.5 & 573.9 \\
\hline Mar. & 11.8 & 953.7 & 949.2 & 845.4 & 441.4 \\
\hline April & 12.6 & 968.4 & 961.9 & 934.0 & 256.1 \\
\hline May & 13.2 & 973.7 & 935.8 & 968.5 & 101.1 \\
\hline June & 13.5 & 974.8 & 912.6 & 974.5 & 024.4 \\
\hline July & 13.4 & 974.8 & 922.9 & 973.2 & 055.0 \\
\hline Aug. & 12.9 & 972.2 & 954.2 & 954.4 & 184.9 \\
\hline Sep. & 12.1 & 962.0 & 962.0 & 888.6 & 368.7 \\
\hline Oct. & 11.4 & 941.6 & 919.8 & 777.4 & 531.3 \\
\hline Nov. & 10.8 & 914.2 & 848.2 & 658.8 & 633.9 \\
\hline Dec. & 10.5 & 897.6 & 806.2 & 599.6 & 668.0 \\
\hline Total & $\mathbf{4 3 8 0}$ & $\mathbf{1 1 3 6 9 . 6}$ & $\mathbf{1 0 8 9 4 . 9}$ & $\mathbf{9 9 3 6 . 4}$ & $\mathbf{4 4 9 0 . 0}$ \\
\hline
\end{tabular}

Tab. 6: The radiation flux incident at 12 noon on different surfaces in Riyadh site.

\begin{tabular}{|c|c|c|c|c|c|}
\hline \multirow{2}{*}{ Month } & \multirow{2}{*}{\begin{tabular}{c}
$\mathbf{h} \mathbf{)})$ \\
\cline { 3 - 6 }
\end{tabular}} & \multicolumn{3}{|c|}{ Solar radiation flux $\left(\mathbf{W h r} / \mathbf{m}^{\mathbf{2}}\right)$} \\
\cline { 3 - 6 } & $\mathbf{I}_{\mathbf{D N}}$ & $\mathbf{I}_{\mathbf{D}}$ & $\mathbf{I}_{\mathbf{D H}}$ & $\mathbf{I}_{\mathbf{D V}}$ \\
\hline Jan. & 10.6 & 906.0 & 828.7 & 631.2 & 649.9 \\
\hline Feb. & 11.2 & 931.9 & 896.4 & 735.8 & 571.9 \\
\hline Mar. & 11.8 & 954.0 & 949.8 & 847.1 & 438.8 \\
\hline April & 12.6 & 968.6 & 961.7 & 934.9 & 253.2 \\
\hline May & 13.2 & 973.8 & 935.0 & 968.8 & 098.0 \\
\hline June & 13.5 & 974.8 & 911.6 & 974.5 & 021.3 \\
\hline July & 13.4 & 974.8 & 921.9 & 973.4 & 051.9 \\
\hline Aug. & 12.9 & 972.3 & 953.7 & 955.1 & 181.9 \\
\hline Sep. & 12.1 & 962.2 & 962.2 & 889.9 & 365.9 \\
\hline Oct. & 11.4 & 942.0 & 920.9 & 779.4 & 529.0 \\
\hline Nov. & 10.8 & 914.9 & 849.9 & 661.2 & 632.2 \\
\hline Dec. & 10.5 & 898.4 & 808.2 & 602.3 & 666.6 \\
\hline Total & 4380 & $\mathbf{1 1 3 7 3 . 6}$ & $\mathbf{1 0 9 0 0 . 0}$ & $\mathbf{9 9 5 3 . 8}$ & $\mathbf{4 4 6 0 . 6}$ \\
\hline
\end{tabular}

Tab. 7: The radiation flux incident at 12 noon on different surfaces in Al-Kharj site.

\begin{tabular}{|c|c|c|c|c|c|}
\hline \multirow{2}{*}{ Month } & \multirow{2}{*}{$\mathbf{S}(\mathbf{h r})$} & \multicolumn{4}{|c|}{ Solar radiation flux $\left(\mathbf{W h r} / \mathbf{m}^{\mathbf{2}}\right)$} \\
\cline { 3 - 6 } & & $\mathbf{I}_{\mathbf{D N}}$ & $\mathbf{I}_{\mathbf{D}}$ & $\mathbf{I}_{\mathbf{D H}}$ & $\mathbf{I}_{\mathbf{D V}}$ \\
\hline Jan. & 10.6 & 907.6 & 832.8 & 637.1 & 646.5 \\
\hline Feb. & 11.2 & 933.0 & 899.3 & 740.8 & 567.2 \\
\hline Mar. & 11.8 & 954.6 & 951.0 & 850.8 & 432.9 \\
\hline April & 12.6 & 968.9 & 961.1 & 937.0 & 246.4 \\
\hline May & 13.2 & 973.9 & 933.1 & 969.6 & 090.9 \\
\hline June & 13.5 & 974.8 & 909.0 & 974.7 & 014.2 \\
\hline July & 13.4 & 974.8 & 919.7 & 973.8 & 044.8 \\
\hline Aug. & 12.9 & 972.5 & 952.5 & 956.6 & 175.0 \\
\hline Sept. & 12.1 & 962.7 & 962.7 & 893.1 & 359.6 \\
\hline Oct. & 11.4 & 943.0 & 923.2 & 784.1 & 523.9 \\
\hline Nov. & 10.8 & 916.3 & 853.7 & 666.9 & 628.4 \\
\hline Dec. & 10.5 & 900.2 & 812.6 & 608.4 & 663.6 \\
\hline Total & $\mathbf{4 3 8 0}$ & $\mathbf{1 1 3 8 2 . 4}$ & $\mathbf{1 0 9 1 1 . 0}$ & $\mathbf{9 9 9 2 . 8}$ & $\mathbf{4 3 9 3 . 4}$ \\
\hline
\end{tabular}

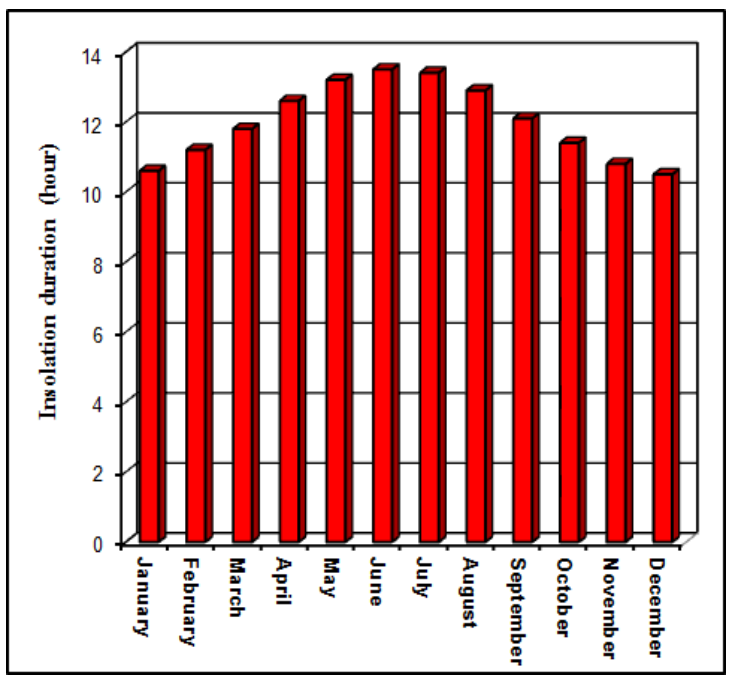

Fig. 2: The variations of insolation duration during the different seasons. 
In general, the minimum insolation duration is 10.5 hours during December, and it increases gradually to reach a maximum of 13.5 hours in June. In total, there are 4380 sun hours per year.

\subsection{Calculation of annual direct solar radiation peak:}

Direct solar radiation is strongly related to direct and vertical solar radiation, the solar incident and the number of hours of insolation duration. This average was calculated at the studied sites at 12 noon everyday as shown in Tables 5, 6 and 7.

The daily direct solar radiation varies relatively from one location to another and from one season to another at the same location as reflected by the daily peak radiation values during each month.

The annual total of direct normal solar intensity peaks in Al-Kharj site at 11382.4 $\mathrm{MWh} / \mathrm{km}^{2}$, with $10911.0 \mathrm{MWh} / \mathrm{km}^{2}$ as the radiation flux incident on titled surfaces, equivalent to $95.9 \%$ of the total of direct normal solar intensity. The radiation flux incident on horizontal surfaces is relatively low with $9992.8 \mathrm{MWh} / \mathrm{km}^{2}$ and the radiation flux incident on vertical surfaces with 4393.4 $\mathrm{MWh} / \mathrm{km}^{2}$, equivalent respectively to $87.8 \%$ and $36.5 \%$ of the total direct normal solar intensity (Fig. 3).

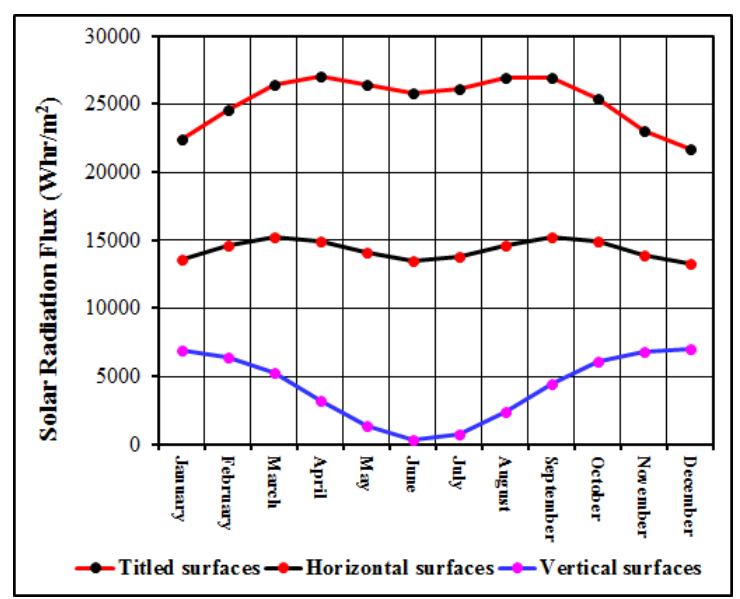

Fig. 3: Monthly average of solar radiation flux incident on different surfaces in Al-Dera'iyah site.

In Riyadh site, the annual total of direct normal solar intensity peaks at 11373.6 $\mathrm{MWh} / \mathrm{km}^{2}$, with $10900.0 \mathrm{MWh} / \mathrm{km}^{2}$ as the radiation flux incident on titled surfaces, equivalent to $95.8 \%$ of the total of direct normal solar intensity. The radiation flux incident on horizontal surfaces is relatively low with $9953.8 \mathrm{MWh} / \mathrm{km}^{2}$ and the radiation flux incident on vertical surfaces with 4460.6 $\mathrm{MWh} / \mathrm{km}^{2}$, equivalent respectively to $91.3 \%$ and $39.2 \%$ of the total direct normal solar intensity (Fig. 4).

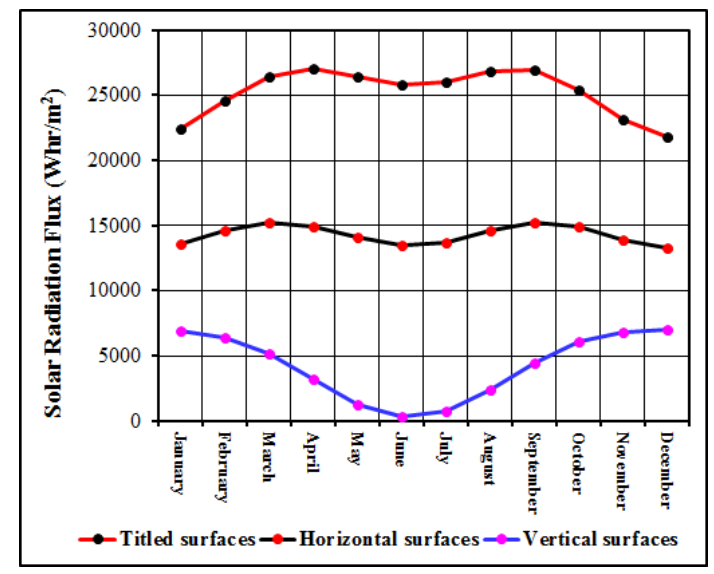

Fig. 4: Monthly average of solar radiation flux incident on different surfaces in Riyadh site.

Also, In Al-Dera'iyah site, the annual total of direct normal solar intensity peaks at $11369.6 \mathrm{MWh} / \mathrm{km}^{2}$, with $10894.9 \mathrm{MWh} / \mathrm{km}^{2}$ as the radiation flux incident on titled surfaces, equivalent to $95.8 \%$ of the total of direct normal solar intensity. The radiation flux incident on horizontal surfaces is relatively low with $9936.4 \mathrm{MWh} / \mathrm{km}^{2}$ and the radiation flux incident on vertical surfaces with 4490.0 $\mathrm{MWh} / \mathrm{km} 2$, equivalent respectively to $87.4 \%$ and $39.5 \%$ of the total direct normal solar intensity (Fig. 5).

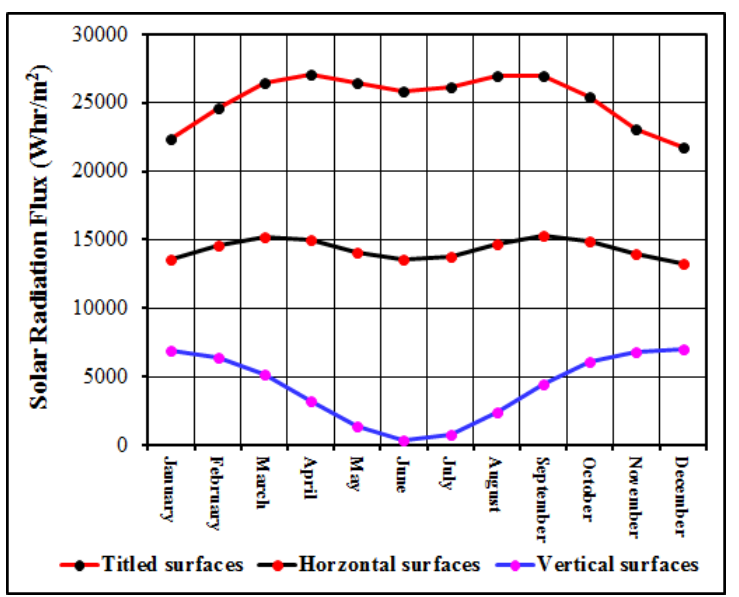

Fig. 5: Monthly average of solar radiation flux incident on different surfaces in Al Kharj site. 


\subsection{Calculation of seasonal direct solar radiation peak:}

The daily solar radiation also varies from one location to another and from one season to the other. So, the direct normal solar radiation intensity is highest at 12 noon during the summer, with $2922.1 \mathrm{MWh} / \mathrm{km}^{2}$ in Al-Kharj, 2921.7 MWh $/ \mathrm{km}^{2}$ in Al-Dera'iyah and 2921.8 $\mathrm{MWh} / \mathrm{km}^{2}$ in Riyadh, equivalent to $25.7 \%$ of the total annual direct normal solar radiation intensity in each location (Fig. 6, 7 and 8).

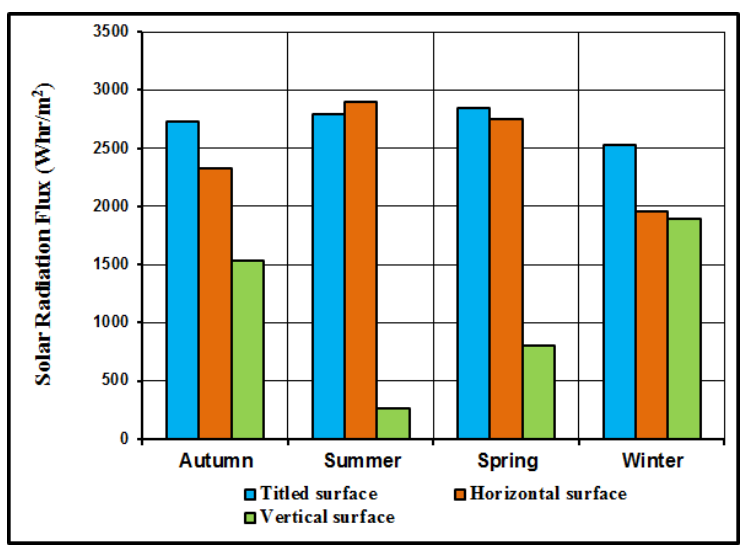

Fig. 6: Seasonal variations of solar radiation flux at 12 noon in Al-Dera'iyah site.

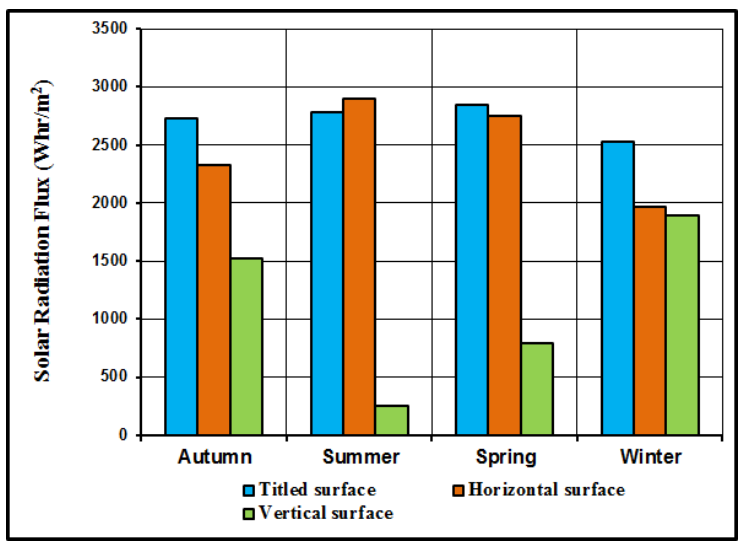

Fig. 7: Seasonal variations of solar radiation flux at 12 noon in Riyadh site.

The direct solar radiation is also highest on the horizontal surfaces during the summer, with 2903.1 MWh $/ \mathrm{km}^{2}$ in Riyadh, 2901.2 MWh $/ \mathrm{km}^{2}$ in Al-Dera'iyah and 2781.3 MWh/ $\mathrm{km}^{2}$ in $\mathrm{Al}$ Kharj, equivalent to $25.5 \%$ of the total annual direct normal solar radiation intensity in each location (Figures 6,7 and 8). This radiation reaches during June a peak of $974.5 \mathrm{MWh} / \mathrm{km}^{2}$ in Riyadh, 974.7 MWh/ $\mathrm{km}^{2}$ in Al-Dera'iyah, equivalent to $33.6 \%$ of the total annual direct normal solar radiation intensity in the summer.

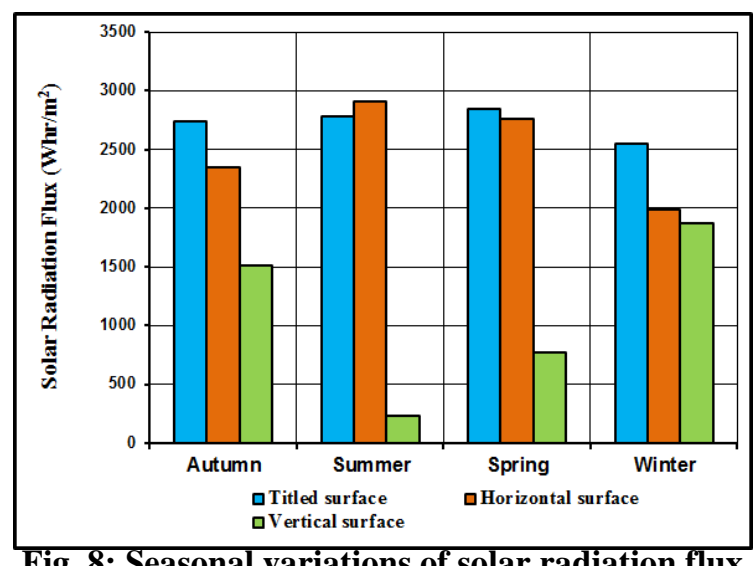

Fig. 8: Seasonal variations of solar radiation flux at 12 noon in Al-Kharj site.

During the spring, the radiation flux incident on horizon surfaces reaches a maximum of $2846.4 \mathrm{MWh} / \mathrm{km}^{2}$ in Riyadh, 2846.9 MWh $/ \mathrm{km}^{2}$ in Al-Dera'iyah and 2845.3 $\mathrm{MWh} / \mathrm{km}^{2}$ in $\mathrm{Al}-\mathrm{Kh}$ arj, equivalent $26.1 \%$ of total annual direct normal solar radiation intensity in each site. However, this radiation reached a peak of $961.7 \mathrm{MWh} / \mathrm{km}^{2}$ in Riyadh, 961.5 $\mathrm{MWh} / \mathrm{km}^{2}$ in Al-Dera'iyah and 961.1 $\mathrm{MWh} / \mathrm{km}^{2}$ in Al-Kharj, equivalent to $33.8 \%$ of the total direct solar radiation during spring.

The spring season is followed by 2733.0 $\mathrm{MWh} / \mathrm{Km}^{2}$ in Riyadh, 2730.0 MWh/ $\mathrm{Km}^{2}$ in AlDera'iyah and 2765.1 MWh/ $\mathrm{Km}^{2}$ in Al-Kharj, equivalent to $25.1 \%$ of the total annual direct normal solar radiation intensity in every site. However, this radiation reached a peak of 962.2 $\mathrm{MWh} / \mathrm{Km}^{2}$ in Riyadh, $962.0 \mathrm{MWh} / \mathrm{Km}^{2}$ in $\mathrm{Al}-$ Dera'iyah and $961.7 \mathrm{MWh} / \mathrm{Km}^{2}$ in Al-Kharj, equivalent to $35.2 \%$ of the total direct solar radiation during autumn.

During the winter, the direct solar radiation is $2533.3 \mathrm{MWh} / \mathrm{Km}^{2}$ in Riyadh, 2528.2 $\mathrm{MWh} / \mathrm{Km}^{2}$ in Al-Dera'iyah and 2544.8 $\mathrm{MWh} / \mathrm{Km}^{2}$ in Al-Kharj, equivalent to $23.2 \%$ of the total annual direct solar radiation in each site. This radiation reaches a peak of 896.4 $\mathrm{MWh} / \mathrm{Km}^{2}$ in Riyadh, 895.1 $\mathrm{MWh} / \mathrm{Km}^{2}$ in AlDera'iyah and 899.3 $\mathrm{MWh} / \mathrm{Km}^{2}$ in Al-Kharj, equivalent to $35.3 \%$ of the total direct solar radiation during winter. 
3.4 Calculate the peak of solar radiation incident on solar panels:

The first photovoltaic cell based on the photovoltaic effect, such as solar radiation, was designed in 1888 by the Russian physicist Aleksander Stoletov (Gevorkian, 2007). In 1954, the high-efficiency solar photovoltaic cell was developed by physicist Daryl Chapin at American Bell Laboratories (APS, 2009).

Solar panel cells technology has become one of the most technologies used in the world to produce electricity from solar energy. The energy of solar panel cells is renewable and clean. It depends on the time of solar insolation, the total direct normal solar radiation intensity and the efficiency of the solar panel cell in converting solar energy into electrical energy.

The solar panel cells vary in size and type , with the dimensions of $164 \mathrm{~cm} \times 99 \mathrm{~cm}$ and $196 \mathrm{~cm} \mathrm{x} 99 \mathrm{~cm}$. The peak of direct solar radiation flux incident on surface of each type was estimated at noon during the various seasons (Tables 8, 9 and 10).

The data of these tables show that extended area of large solar panels $(196 \mathrm{~cm} \mathrm{x} 99 \mathrm{~cm})$ increases the direct solar radiation flux incident on titled surface during the seasons of winter, spring and autumn, and the direct solar radiation flux incident on horizontal surface during the summer. The difference percentage of this increase between the two types of solar panel cells is $16.3 \%$.

The size of the photovoltaic matrix producing electricity depends on the size of the solar panels converting the solar energy into electrical energy. So, the peak of direct solar radiation flux incident at noon on the large panels is ranged from $5633.1 \mathrm{Wh} / \mathrm{m}^{2}$ to $15742.0 \mathrm{Wh} / \mathrm{m}^{2}$ on large solar panel cells. However, the peak of direct solar radiation flux incident at noon on the small panels is ranged from $4713.4 \mathrm{Wh} / \mathrm{m}^{2}$ on horizontal small panel and $13,171.9 \mathrm{Wh} / \mathrm{m}^{2}$ on titled panel.

Tab. 8: Peak of Solar Direct flux $\left(\mathrm{Whr}^{2} \mathrm{~m}^{2}\right)$ of Solar Panel at 12 noon in Al-Dera'iyah site.

\begin{tabular}{|c|c|c|c|c|c|c|}
\hline \multirow{3}{*}{ Month } & \multirow{2}{*}{\multicolumn{2}{|c|}{$\begin{array}{l}\text { Peak of Solar Direct } \\
\text { Flux }\left(\mathrm{Whr} / \mathrm{m}^{2}\right)\end{array}$}} & \multicolumn{4}{|c|}{ Solar panel dimensions } \\
\hline & & & \multicolumn{2}{|c|}{$196 \mathrm{~cm} \times 99 \mathrm{~cm}$} & \multicolumn{2}{|c|}{$164 \mathrm{~cm} \times 99 \mathrm{~cm}$} \\
\hline & $\begin{array}{c}\text { Titled } \\
\text { surface }\end{array}$ & $\begin{array}{c}\text { Horizontal } \\
\text { surface }\end{array}$ & $\begin{array}{c}\text { Horizontal } \\
\text { surface }\end{array}$ & $\begin{array}{c}\text { Titled } \\
\text { surface }\end{array}$ & $\begin{array}{c}\text { Horizontal } \\
\text { surface }\end{array}$ & $\begin{array}{c}\text { Titled } \\
\text { surface }\end{array}$ \\
\hline January & 848.2 & --- & --- & 1645.9 & --- & 1377.2 \\
\hline February & 806.2 & --- & --- & 1564.4 & --- & 1308.9 \\
\hline March & 826.9 & --- & --- & 1604.5 & --- & 1342.6 \\
\hline April & 895.1 & --- & --- & 1736.9 & --- & 1453.3 \\
\hline May & 949.2 & --- & --- & 1841.8 & --- & 1541.1 \\
\hline June & 961.9 & --- & --- & 1866.4 & --- & 1561.7 \\
\hline July & --- & 935.8 & 1815.8 & --- & 1519.4 & --- \\
\hline August & --- & 974.5 & 1890.8 & --- & 1582.1 & --- \\
\hline September & --- & 973.2 & 1888.4 & --- & 1580.1 & --- \\
\hline October & 954.4 & --- & --- & 1852.0 & --- & 1549.6 \\
\hline November & 962.0 & --- & --- & 1866.6 & --- & 1561.8 \\
\hline December & 919.8 & --- & --- & 1784.8 & --- & 1493.4 \\
\hline Total & 8123.7 & 2883.5 & 5595.1 & 15763.3 & 4681.6 & 13189.7 \\
\hline
\end{tabular}


Tab. 9: Peak of Solar Direct Flux $\left(\mathrm{Whr} / \mathrm{m}^{2}\right)$ of Solar Panel at 12 noon in Riyadh site.

\begin{tabular}{|c|c|c|c|c|c|c|}
\hline \multirow{3}{*}{ Month } & \multirow{2}{*}{\multicolumn{2}{|c|}{$\begin{array}{l}\text { Peak of Solar Direct } \\
\text { Flux }\left(\mathrm{Whr} / \mathrm{m}^{2}\right)\end{array}$}} & \multicolumn{4}{|c|}{ Solar panel dimensions } \\
\hline & & & \multicolumn{2}{|c|}{$196 \mathrm{~cm} \times 99 \mathrm{~cm}$} & \multicolumn{2}{|c|}{$164 \mathrm{~cm} \times 99 \mathrm{~cm}$} \\
\hline & $\begin{array}{c}\text { Titled } \\
\text { surface }\end{array}$ & $\begin{array}{c}\text { Horizontal } \\
\text { surface }\end{array}$ & $\begin{array}{c}\text { Horizontal } \\
\text { surface }\end{array}$ & $\begin{array}{c}\text { Titled } \\
\text { surface }\end{array}$ & $\begin{array}{c}\text { Horizontal } \\
\text { surface }\end{array}$ & $\begin{array}{c}\text { Titled } \\
\text { surface }\end{array}$ \\
\hline January & 808.2 & --- & --- & 1568.2 & --- & 1312.2 \\
\hline February & 828.7 & --- & --- & 1608.1 & --- & 1345.5 \\
\hline March & 896.4 & --- & --- & 1739.4 & --- & 1455.4 \\
\hline April & 949.8 & --- & --- & 1842.9 & --- & 1542.0 \\
\hline May & 961.7 & --- & --- & 1866.0 & --- & 1561.4 \\
\hline June & 935.0 & --- & --- & 1814.3 & --- & 1518.1 \\
\hline July & --- & 974.5 & 1891.0 & --- & 1582.3 & --- \\
\hline August & --- & 973.4 & 1888.8 & --- & 1580.4 & --- \\
\hline September & --- & 955.1 & 1853.3 & --- & 1550.7 & --- \\
\hline October & 962.2 & --- & --- & 1867.1 & --- & 1562.2 \\
\hline November & 920.9 & --- & --- & 1786.9 & --- & 1495.1 \\
\hline December & 849.9 & --- & --- & 1649.2 & --- & 1380.0 \\
\hline Total & 8112.8 & 2903.1 & 5633.1 & 15742.0 & 4713.4 & 13171.9 \\
\hline
\end{tabular}

Tab. 10: Peak of Solar Direct Flux $\left(\mathrm{Whr} / \mathrm{m}^{2}\right)$ of Solar Panel at 12 noon in Al-Kharj site.

\begin{tabular}{|c|c|c|c|c|c|c|}
\hline \multirow{3}{*}{ Month } & \multirow{2}{*}{\multicolumn{2}{|c|}{$\begin{array}{l}\text { Peak of Solar Direct Flux } \\
\left(\mathrm{Whr} / \mathrm{m}^{2}\right)\end{array}$}} & \multicolumn{4}{|c|}{ Solar panel dimensions } \\
\hline & & & \multicolumn{2}{|c|}{$196 \mathrm{~cm} \times 99 \mathrm{~cm}$} & \multicolumn{2}{|c|}{$164 \mathrm{~cm} \times 99 \mathrm{~cm}$} \\
\hline & $\begin{array}{c}\text { Titled } \\
\text { surface }\end{array}$ & $\begin{array}{l}\text { Horizontal } \\
\text { surface }\end{array}$ & $\begin{array}{c}\text { Horizontal } \\
\text { surface }\end{array}$ & $\begin{array}{c}\text { Titled } \\
\text { surface }\end{array}$ & $\begin{array}{c}\text { Horizontal } \\
\text { surface }\end{array}$ & $\begin{array}{c}\text { Titled } \\
\text { surface }\end{array}$ \\
\hline January & 812.6 & --- & --- & 1576.8 & --- & 1319.4 \\
\hline February & 832.8 & --- & --- & 1616.1 & --- & 1352.2 \\
\hline March & 899.3 & --- & --- & 1745.0 & --- & 1460.1 \\
\hline April & 951.0 & -- & --- & 1845.4 & --- & 1544.1 \\
\hline May & 961.1 & --- & --- & 1865.0 & --- & 1560.5 \\
\hline June & 933.1 & --- & --- & 1810.6 & --- & 1515.0 \\
\hline July & --- & 974.7 & 1891.3 & --- & 1582.5 & --- \\
\hline August & --- & 973.8 & 1889.6 & --- & 1581.1 & --- \\
\hline September & --- & 956.6 & 1856.3 & --- & 1553.2 & --- \\
\hline October & 962.7 & --- & --- & 1868.1 & --- & 1563.1 \\
\hline November & 923.2 & --- & --- & 1791.4 & --- & 1498.9 \\
\hline December & 853.7 & --- & --- & 1656.6 & --- & 1386.1 \\
\hline Total & 8129.7 & 2905.1 & 5637.1 & 15774.9 & 4716.8 & 13199.4 \\
\hline
\end{tabular}




\section{CONCLUSION:}

The study had showed that the three studied sites have duration of sunrise of more than 10 hours/day with an annual total of 4380 hours/year of solar insolation and 2.91 $\mathrm{MWh} / \mathrm{km}^{2}$ of direct radiation flux incident on titled surfaces and $1.13 \mathrm{MWh} / \mathrm{km}^{2}$ of direct radiation flux incident on the horizontal surfaces of the studied sites. This quantity of solar radiation is an important source of renewable energy that can be exploited in the production of electric power in Riyadh.

Also, this study was able to estimate the daily average of direct solar radiation on the titled, horizontal and vertical surfaces in order to determine which is most able to convert the solar energy incident to the electric power. The calculation of direct radiation flux incident on the study area showed that:

1. The titled surfaces are the best surfaces to save the solar energy with a total of 131204.3 MW/hr/ $/ \mathrm{km}^{2}$ in Riyadh and 131161.1 MW/hr/ $/ \mathrm{km}^{2}$ in Al-Dera'iyah and 131276.4 MW / hr / km 2in Al-Kharj.

2. During different seasons, he titled surfaces are the best surfaces to save the solar energy, except the summer. They can save a total of direct radiation flux up to 2847 $\mathrm{MW} / \mathrm{hr} / \mathrm{km}^{2}$ during the spring, to 2740 $\mathrm{MW} / \mathrm{hr} / \mathrm{km}^{2}$ during the autumn and to 2545 $\mathrm{MW} / \mathrm{km}^{2}$ during the winter.

3. During the summer, the horizontal surfaces are the best surfaces for saving the direct radiation flux with $2905 \mathrm{MWh} / \mathrm{km}^{2}$.

4. During the different seasons except the summer, the titled surfaces are the best surfaces for saving the direct radiation flux, can rising at 12 noon to $949.0 \mathrm{MW} / \mathrm{hr} / \mathrm{km}^{2}$ during spring, $913.2 \mathrm{MW} / \mathrm{hr} / \mathrm{km}^{2}$ during autumn and $848.3 \mathrm{MW} / \mathrm{hr} / \mathrm{km}^{2}$ during winter.

5. The horizontal surfaces are the best surfaces to save the solar energy during the summer a total of direct radiation flux up to 968.4 MW/hr/ $/ \mathrm{km}^{2}$ at 12 noon.
6. Al-Kharj area is considered the best site for providing solar energy during the year. It has the highest daily and seasonal average of direct radiation flux incident on titled surfaces. It is possible to invest in this region to design photovoltaic matrices based on solar cell technology to product the electric power.

7. There is a great potential for producing electric power in the sites studied using the technology of large solar panels with $16.3 \%$ more than the small solar panels, especially in Al-Kharj area.

\section{REFERENCES:}

Ashrae, (2011): ASHRAE Handbook, 1985. Fundamentals, American Society of Heating, Refregering and Air-Conditioning Engineers, Inc.: Atlanta, GA, USA.

APS, (2009): Bell Labs Demonstrates the First: Practical Silicon Solar Cells, APS News, American Physical Society 18 (4).

Cooper, P.I. (1969): The absorption of Radiation in Solar Stills, Sol. Energy 12 (3): 333-346.

Duffie, A. and Backman, W.A. (2013): Solar engineering thermal processes, John Wiley and Sons, New York, USA.

Gevorkian, P. (2007): Sustainable Energy Systems Engineering: The Complete Green Building Design Resource, McGraw Hill Professional, ISBN 978-0-07-147359-0.

Lunde, P.J. (1980): Solar thermal engineering: Space heating and hot water systems. United States.

Messenger, R.A. and Vente, J. (2003): Photovoltaic systems engineering, CRC Press, Boca Rato, U.S.A. 


\section{الأستفادة من الإشعاع الشمسي \\ كطاقة بليلة في منطقة الرياض بالمملكة العربية السعودية \\ مطيرة كويتم المطيري \\ جامعة الأميرة نورة بنت عبد الرحمن- الرياض- المملكة العربية السعودية}

الملخص

تتميز المو اقع المدروسـة في منطقة الريـاض بوفرة كبيرة من الموارد الطبيعيـة و البشـرية على مدار

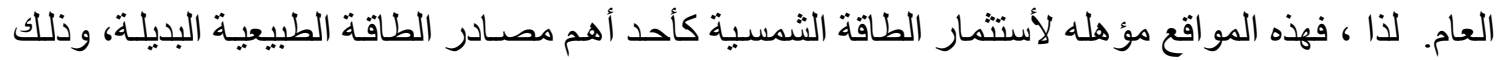

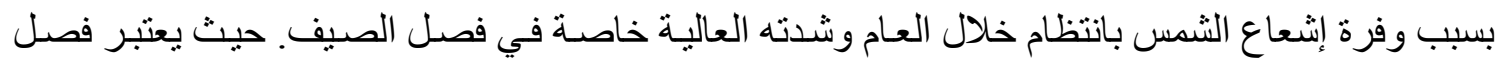

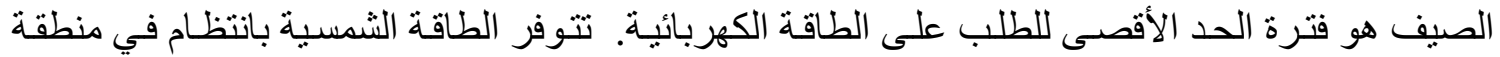

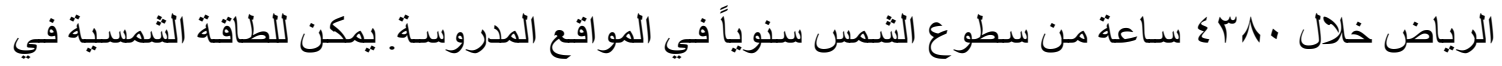

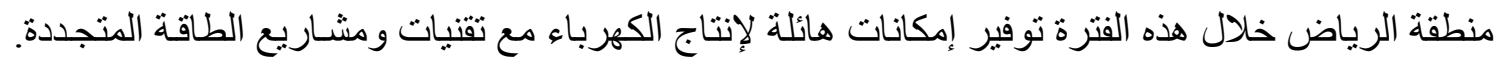

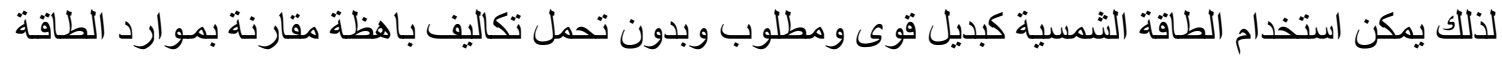

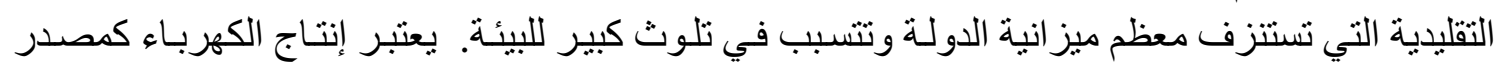

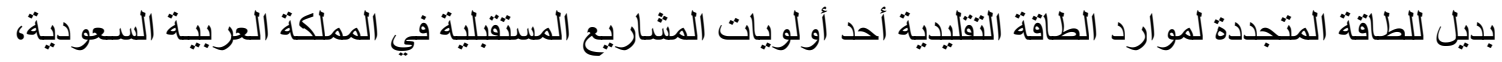

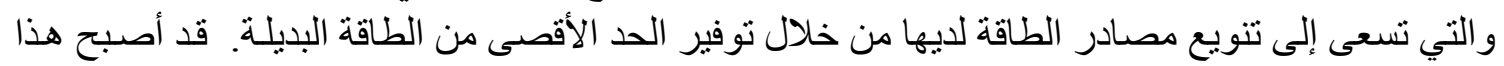

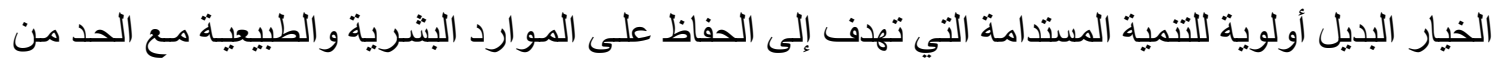

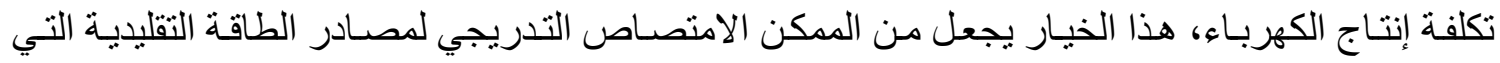
تلوث البيئة وتهدد باستنز افها. 